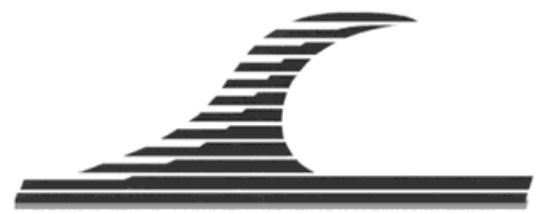

\title{
Le complexe dunaire quaternaire du Jorf Lasfar (Meseta côtière occidentale marocaine) : lithostratigraphie, sédimentologie et néotectonique
}

\section{Mohamed OUADIA $^{1}$, M'Hamed ABERKAN ${ }^{2}$, Khadija ABOUMARIA $^{3}$}

1. Université Chouaïb Doukkali, Faculté des Sciences, Département des Sciences de la Terre, B.P. 20, El Jadida, 24000, Maroc.

mhouadia@yahoo.fr

2. Université Mohammed V, Faculté des Sciences, Département des Sciences de la Terre, B.P. 1014, Rabat, Maroc.

ma_aberkan@yahoo.fr

3. Université Abdelmalek Sâadi, Faculté des Sciences et Techniques, Département des Sciences de la Terre, Tanger, Maroc.

kaboumaria@caramail.com

\section{Résumé :}

Le complexe dunaire quaternaire du Jorf Lasfar, creusé transversalement lors de l’installation du complexe industriel Maroc Phosphore III et IV, révèle la coupe la plus développée et la plus détaillée dans la région. Il est formé de calcarénites dans lesquelles s'intercalent des paléosols rouges. Il reflète une période climatique quaternaire sèche, probablement soltanienne, entrecoupée par des phases humides et tempérées. Les sables biocalcarénitiques formant ce complexe dunaire résultent d'une reprise de sédiments de plage dont les éléments terrigènes proviennent de plusieurs sources telles que le Moyen Atlas, la Méséta côtière et le massif des Réhamna. Le complexe dunaire de Jorf Lasfar est affecté par une néotectonique qui prouve que ce secteur de la Méséta côtière marocaine n'a pas été stable au cours du Quaternaire, contrairement à ce qui a été admis dans les travaux antérieurs.

Soumis le 14 novembre 2014, accepté le 17 juillet 2015, en ligne le 14 décembre 2015.

La seule version examinée est celle écrite en français. La ou les autres versions n'étant pas examinées par le comité de rédaction de la revue, sont donc publiées sous l'entière responsabilité du ou des auteurs.

A TRANSLATED VERSION IN ENGLISH IS AVAILABLE ONLINE

Pour citer cet article :

OUADIA M., ABERKAN M., ABOUMARIA K. (2015). Le complexe dunaire quaternaire du Jorf Lasfar (Meseta côtière occidentale marocaine) : lithostratigraphie, sédimentologie et néotectonique. Revue Paralia, Vol. 8, pp n02.1-n02.14.

DOI: http://dx.doi.org/10.5150/revue-paralia.2015.n02 


\section{Introduction}

Au Sud d'El Jadida, dans le secteur du Jorf Lasfar, les cordons dunaires côtiers n'ont pas fait l'objet d'une étude géologique détaillée antérieure. Les travaux réalisés par GIGOUT (1947), GIGOUT (1951) et AKIL (1990) intéressent les calcarénites quaternaires de la région des Doukkala mais le complexe du Jorf Lasfar n'a pas été étudié. Avec la construction du port du Jorf Lasfar et du complexe industriel Maroc Phosphore III et IV des coupes nouvelles sont apparues et nous ont permis d'étudier en détail ce complexe côtier. Cette note se fixe pour but de mettre en évidence l'origine et les dynamiques responsables de la mise en place de ces calcarénites. En outre, elle décrit, dans cette zone de la Méséta côtière marocaine réputée stable au cours du Quaternaire, une activité néotectonique. Elle est matérialisée par des failles normales et inverses qui ont affecté en particulier le paléosol 1 et la dune 2 de ce complexe dunaire.

\section{Situation géographique}

La zone du Jorf Lasfar est située juste au Sud du complexe industriel Maroc Phosphore III et IV d'El Jadida. Ses coordonnées Lambert sont $\mathrm{X}=284,5 \mathrm{~km}$ et $\mathrm{Y}=198,5 \mathrm{~km}$ (voir figure 1).
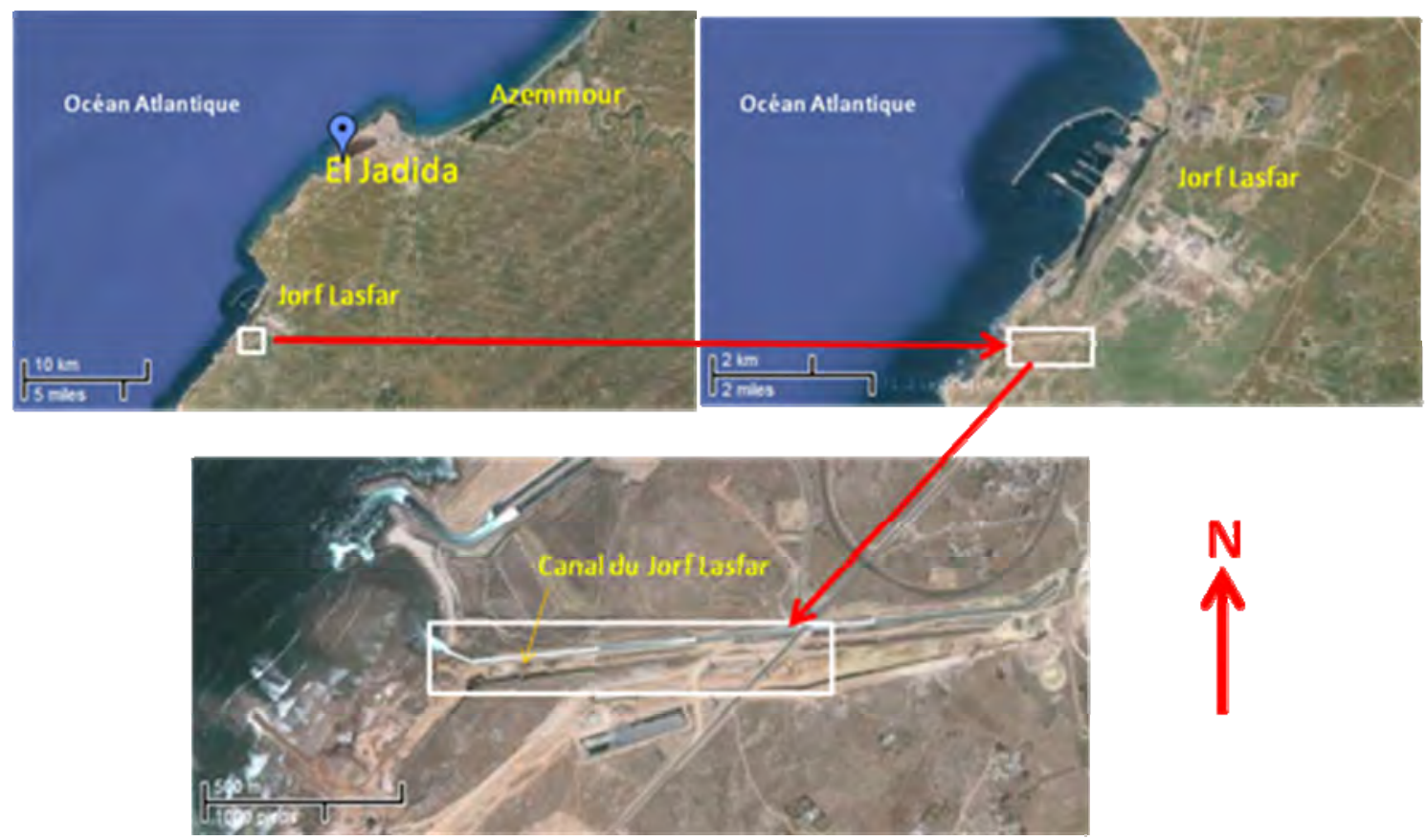

Figure 1. Situation géographique de la zone étudiée

(Source des Photos : Google Earth).

\section{Lithostratigraphie du complexe dunaire du Jorf Lasfar}

Du bas vers le haut on distingue (voir figure 2) : 
3.1 L'édifice dunaire est une biocalcarénite consolidée à grandes stratifications entrecroisées, avec des débris de coquilles marines et des coquilles terrestres bien conservées, au sommet. Son épaisseur maximale est de $3 \mathrm{~m}$ et sa couleur est jaune à la base et rouge brique clair au sommet. A son sommet, on note la présence de quelques traces de racines fossilisées en position de vie $(10 \mathrm{~cm}$ de longueur maximale) et quelques nodules calcaires (2 à $10 \mathrm{~cm}$ de diamètre). La limite inférieure de cette unité n'est pas visible.

3.2 Le paléosol 1 représente l'horizon inférieur d'un profil pédologique de couleur rougeâtre dont les horizons supérieurs sont érodés. Il a 2,5 m d'épaisseur au maximum. Il renferme de nombreuses coquilles brisées ou entières de gastéropodes pulmonés. Les racines sont abondantes de longueur maximale pouvant atteindre $20 \mathrm{~cm}$ et certaines se prolongent dans l'édifice dunaire sous-jacent. Elles sont associées à des nodules carbonatées (2 à $8 \mathrm{~cm}$ de diamètre), en particulier à la base de ce paléosol. La structure de ce dernier est polyédrique et sa limite inférieure est progressive.

3.3 L’édifice dunaire 2 est une biocalcarénite consolidée de $4 \mathrm{~m}$ d'épaisseur maximale et de couleur jaune clair à la base et rouge brique clair au sommet. Sa limite inférieure est nette. Comme dans le cas de l'édifice dunaire 1, on note la présence des débris de coquilles marines à la base et des coquilles terrestres relativement bien conservées ainsi que des nodules calcaires jusqu'à $5 \mathrm{~cm}$ de diamètre au sommet. La stratification entrecroisée est moins visible que dans l'édifice dunaire 1.

3.4 Le paléosol 2 de couleur moins rougeâtre est moins riche en concrétions carbonatées que le paléosol 1 atteint 0,8 m d'épaisseur. Sa limite inférieure est graduelle. Ce paléosol représente aussi un profil pédologique érodé. Il a une structure prismatique et renferme de nombreuses coquilles brisées ou entières de gastéropodes pulmonés.

3.5 L’édifice dunaire 3 est une biocalcarénite consolidée, de 2,7 m d'épaisseur maximale et de couleur jaune clair à la base et rouge clair au sommet. A son sommet, on note la présence de quelques racines (longueur maximale de $10 \mathrm{~cm}$ ) et quelques nodules calcaires de 2 à $10 \mathrm{~cm}$ de diamètre. Sa limite inférieure est nette mais la stratification oblique est peu visible. On observe des débris de coquilles marines à la base et des coquilles terrestres relativement bien conservées au sommet.

3.6 Le paléosol 3 de couleur rougeâtre, atteint $0,7 \mathrm{~m}$ d'épaisseur. Les racines sont abondantes et certaines se prolongent dans l'édifice dunaire sous-jacent. Leur longueur maximale atteint $12 \mathrm{~cm}$ et elles sont associées à des concrétions carbonatées (2 à $5 \mathrm{~cm}$ de diamètre). La limite inférieure de ce paléosol est progressive. Sa structure est 
faiblement polyédrique et il renferme de nombreuses coquilles, souvent entières, de gastéropodes pulmonés.

3.7 L’édifice dunaire 4 correspond à une biocalcarénite moins consolidée, de $2 \mathrm{~m}$ d'épaisseur maximale et de couleur jaune clair à la base et rouge clair au sommet. Les débris de coquilles terrestres sont moins fréquents que dans les édifices dunaires précédents. A son sommet, on note seulement la présence de quelques nodules calcaires de $3 \mathrm{~cm}$ de diamètre en moyenne.

3.8 Le paléosol 4 est épais au maximum de $0,5 \mathrm{~m}$, de couleur rougeâtre et peut être divisé en deux horizons par une croûte calcaire mince (environ $1 \mathrm{~cm}$ d'épaisseur). Il renferme des coquilles souvent brisées de gastéropodes pulmonés et sa limite inférieure est indistincte.

3.9 L'édifice dunaire 5 est constitué par un sable biocalcarénite de couleur beige clair, non consolidé mais fixé par la végétation actuelle. Sa limite avec le paléosol 4 est nette. Les débris de coquilles terrestres sont peu fréquents et très petits. Son épaisseur maximale atteint $0,8 \mathrm{~m}$. Il est surmonté par une croûte calcaire discontinue d'environ 3 cm d'épaisseur.

\subsection{Un sol brun, sub-actuel}

Il est épais d'environ 0,5 m et de couleur brun-rouge. Sa limite inférieure est brutale. Il renferme quelques cailloux de croûtes calcaires et des débris de coquilles de pulmonés terrestres.
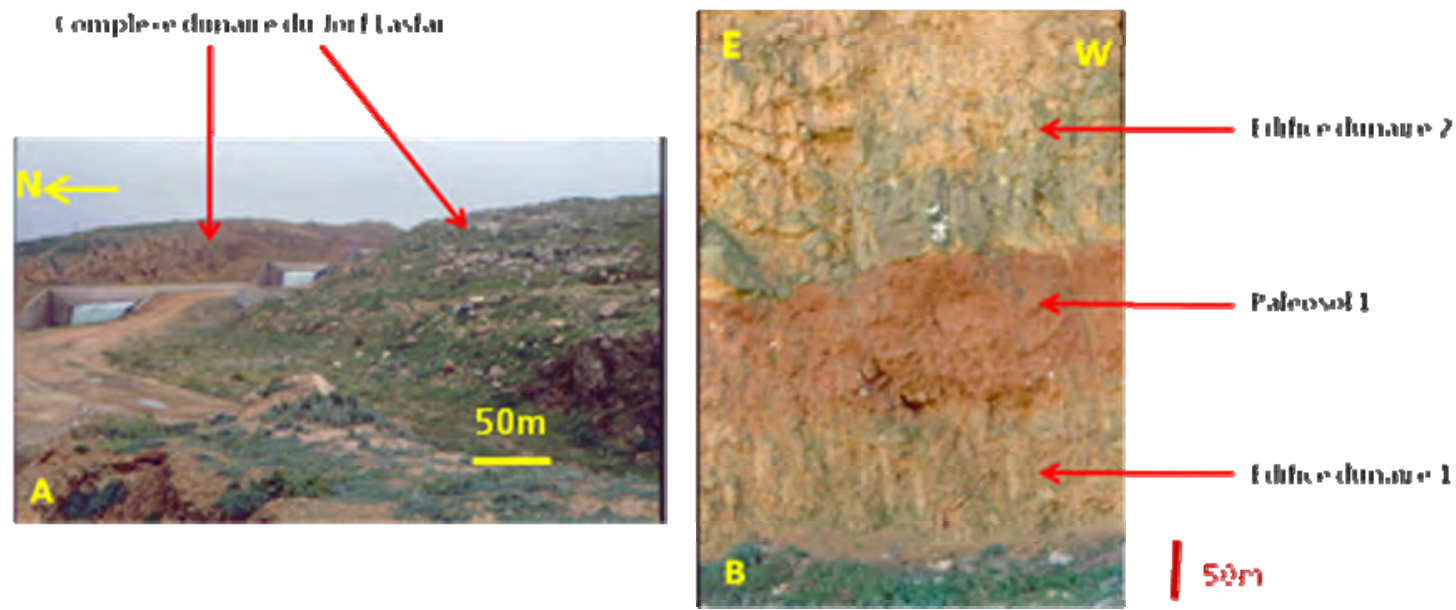

Figure 2. Lithostratigraphie du complexe dunaire du Jorf Lasfar. 


\section{Sédimentologie}

\subsection{Microfaciès}

L'étude microfaciologique des biocalcarénites montre une richesse particulière en bioclastes de lamellibranches, associés à des débris de gastéropodes et d'échinodermes remaniés (tests concassés), dans des dépôts de plage. Ils constituent environ 60\% des éléments figurés. Les éléments terrigènes sont constitués de grains de quartz (plus de $30 \%$ ) et de fragments de roches associés à quelques minéraux lourds et plagioclases. Le ciment carbonaté se présente sous forme de ménisques aux points de contact entre les éléments témoignant d'une cimentation précoce en zone vadose (DUNHAM, 1969). Le ciment tardif correspond à une mosaïque de microsparites colmatant les vides intergranulaires. Au sommet, le ciment n'est que partiellement conservé, affecté par une dissolution due aux circulations vadoses modernes. La dissolution affecte aussi bien les bioclastes que le ciment, entraînant la formation de vides vacuolaires plus importants vers le sommet qu'à la base. La structure des bioclastes est partiellement conservée, malgré une recristallisation partielle (en particulier les fragments de lamellibranches) et un remplacement par des cristaux microsparitiques et sparitiques drusiques qui caractérisent une diagenèse en milieu continental (PURSER, 1980) (voir figure 3A).

$\mathrm{Au}$ niveau des paléosols, on note une abondance de gastéropodes terrestres généralement mal conservés. Les éléments carbonatés y compris les pellets, présentent une recristallisation en cristaux micritiques à microsparitiques. La fraction quartzeuse, très abondante, les plagioclases souvent altérés et les minéraux opaques sont souvent recouverts d'une pellicule ferrugineuse. Les éléments du squelette pédologique sont entourés par un revêtement limono-argileux témoignant d'un apport par lessivage, à partir des horizons supérieurs, des produits d'altération. Les vides résiduels sont colmatés partiellement ou complètement par de la microsparite (voir figure 3B).

\subsection{Granulométrie}

Le tamisage (colonne de tamis de type AFNOR) du matériel d'édifices dunaires montre qu'il est riche en sable par rapport aux limons et aux argiles, avec un pourcentage qui varie entre 22 et 54\%. La valeur de la médiane, comprise entre 102 et $128 \mu \mathrm{m}$, correspond à des sables fins. Les valeurs faibles de l'indice de distribution $[C d=(Q 1-$ Q3)/2] qui varie entre 20 et $40 \mu \mathrm{m}$ (voir tableau 1) et celle du Sorting index $\left[\mathrm{So}=(\mathrm{Q} 1 / \mathrm{Q} 3)^{1 / 2}\right]$ qui est comprise entre 1,24 et 1,35 indiquent que les sédiments sont bien triés. L'indice de Skewness Sk, qui oscille entre 0,71 et 0,98, montre que le tri des sédiments a privilégié les particules les plus grossières (voir tableau 1). 


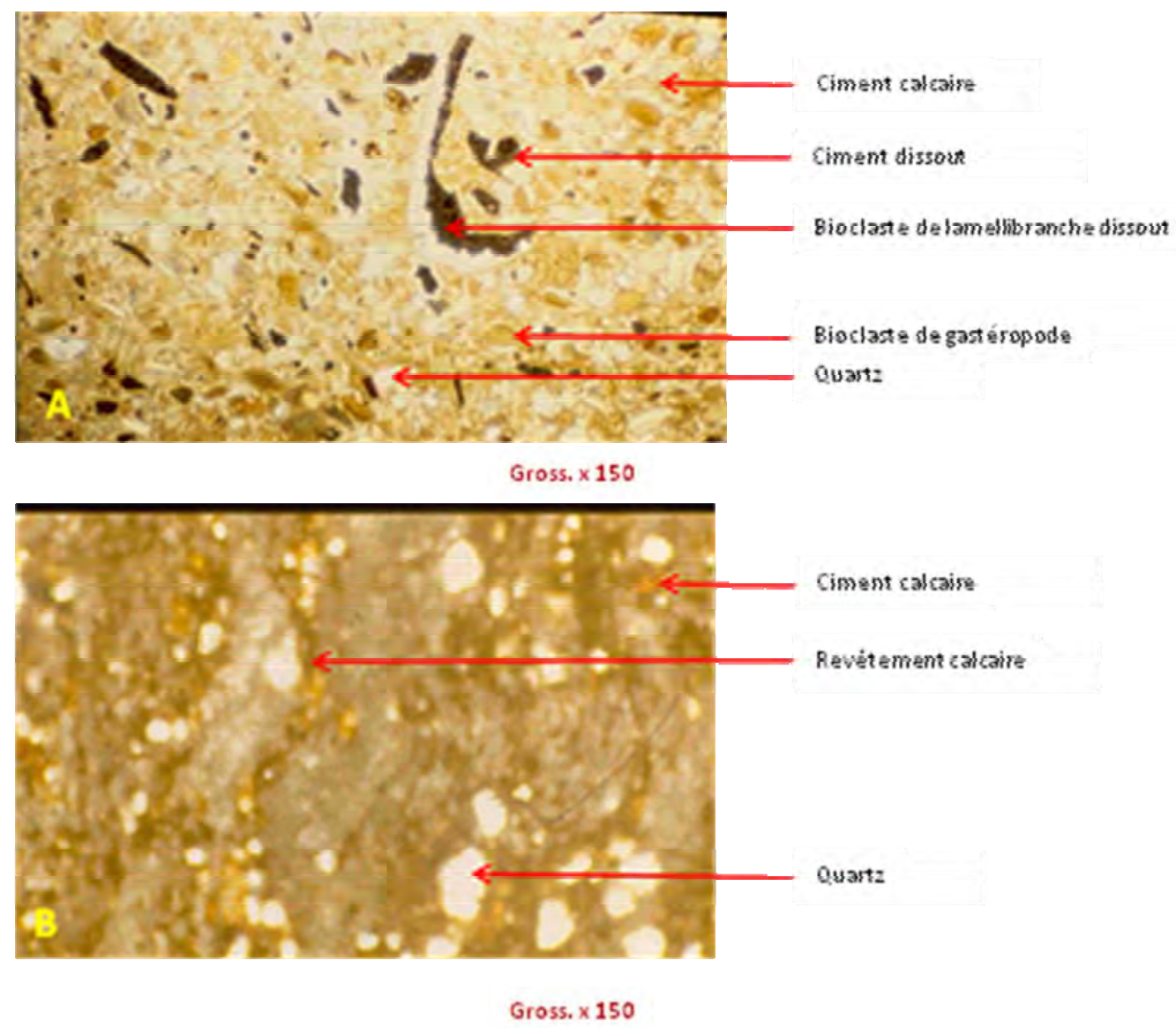

Figure 3: Microfaciès de l'édifice dunaire 1 (échantillon d1-s) (A) et du paléosol (échantillon S1-s) (B).

\subsection{Morphoscopie des grains de quartz}

Après attaque à l'acide chlorhydrique, les grains de quartz observés à la loupe binoculaire, sont de couleur jaunâtre pour les calcarénites dunaires et rose, parfois rouge, pour les paléosols. Cette différence de couleur est due à la présence d'argiles et à la fine pellicule d'oxydes de Fe et de Mn qui recouvre les grains de quartz à l'intérieur des paléosols. Dans les édifices dunaires, les grains de quartz dominants sont ronds mats (41 à 62\%), émoussés luisants (26 à 42\%) et non usés (12 à 25\%) (voir tableau 1). Des types intermédiaires de grains de quartz ont été observés en particulier dans l'édifice dunaire 1 et 2 . Il s'agit de ronds luisants et d'émoussés mats avec des pourcentages ne dépassant pas 15\%. La présence de ces deux types de grains de quartz témoigne d'une interaction entre les dynamique marine littorale et éolienne. La même remarque a été faite sur les grains du quartz issus des dunes littorales quaternaires du Rharb (ABERKAN \& LEGIGAN, 1984; ABERKAN, 1989), de Larache (ADIL \& ABERKAN,1993 ; ADIL, 1996) et de Casablanca (ZANNIBY, 1997). 


\subsection{Minéralogie}

a) Minéraux lourds. La teneur pondérale des minéraux lourds des calcarénites dunaires du Jorf Lasfar est relativement faible, elle est comprise entre 0,07\% (édifice dunaire 1) et $1,92 \%$ (édifice dunaire 3 ) témoignant de la pauvreté des sources de minéraux lourds. Ces derniers sont dominés par la hornblende (4 à 43\%) qui proviendrait, en partie, des formations métamorphiques du massif des Réhamna. La présence du pyroxène s'expliquerait par le volcanisme basaltique du Moyen Atlas et de la Méséta côtière drainés par l'Oued Oum Rbia ainsi que par la dérive littorale de direction N-S (OUADIA \& ABERKAN, 1996). Trois origines de minéraux lourds sont possibles: massif des Réhamna, Moyen Atlas et Méséta côtière. L'indice de maturité des minéraux lourds ( $\%$ tourmaline $+\%$ zircon $+\%$ rutile $+\%$ anatase $+\%$ brookite $) /$ $\%$ autres minéraux lourds transparents) est légèrement élevé. Il oscille entre 0,01 enregistré au niveau de l'édifice dunaire 4 et 0,43 enregistré au niveau du paléosol 1 et témoigne d'une altération des minéraux fragiles lors de la formation des paléosols.

b) Minéraux argileux. Le cortège minéralogique de minéraux argileux échantillonnés dans les paléosols est dominé par l'illite (40\%), les smectites (25\%), la chlorite (15\%) et la kaolinite (5\%) associée à des inter-stratifiés (illite-smectite et smectitechlorite (15\%)). Le degré de cristallinité des minéraux argileux est en général faible sauf pour le cas de l'illite qui présente des pics relativement aigus. Ces argiles sont essentiellement héritées (ABERKAN, 1989 ; ABOUMARIA, 1993).

4.5 Caractéristiques physiques et chimiques des édifices dunaires et des paléosols du Jorf Lasfar

a) Le pH: Le pH des formations quaternaires du Jorf Lasfar est légèrement basique. Ses valeurs varient entre 7,6 dans le paléosol 2 et 8,37 dans l'édifice dunaire 1 (voir tableau 1). Le caractère basique de ces formations est en relation, au moins en partie, avec la forte teneur de ces dernières en carbonate de calcium (plus de $70 \%$ dans les édifices dunaires). Les différences de $\mathrm{pH}$ entre les édifices dunaires et les paléosols résulteraient de leur teneur en $\mathrm{CaCO}_{3}$. En effet, on constate que la valeur du $\mathrm{pH}$ la moins basique est celle des paléosols dont le taux des carbonates est relativement faible ( $17 \%$ au maximum).

b) La matière organique. Ses teneurs sont faibles et varient entre 0,02\% (édifice dunaire 1) et $0,48 \%$ (sommet du paléosol 2). L'augmentation relative de la teneur en matière organique vers le sommet du complexe dunaire du Jorf Lasfar est probablement en rapport avec l'installation de la végétation actuelle (voir tableau 1).

c) Le phosphore assimilable: Le taux du phosphore assimilable montre des valeurs oscillant entre $0,14 \%$ au niveau de l'édifice dunaire 4 et $0,72 \%$ à la base du paléosol 1 (voir tableau 1). Les valeurs relativement élevées du phosphore assimilable au niveau des paléosols s'expliquerait par la relative richesse de ces derniers en matière organique, traduisant une activité biologique, qui tend à influencer la teneur en $\mathrm{P}_{2} \mathrm{O}_{5}$. 
d) Le fer total. Les teneurs en fer total sont variables. Elles sont plus élevées dans le paléosol 1 (2,11\%). Au-delà, dans tous les niveaux sus-jacents, cette teneur diminue et atteint une valeur minimale de l'ordre de 0,43\% dans l'édifice dunaire 3 et dans le paléosol 3. La teneur relativement forte du fer dans le paléosol 1 serait en relation étroite avec les phénomènes de libération de fer par des minéraux primaires en raison de la rubéfaction marquée de ce niveau. Les paléosols sont plus riches en fer que les édifices dunaires en raison de la concentration relative du fer et des argiles par la décarbonatation des calcarénites dunaires (ABERKAN, 1989), (voir tableau 1).

Tableau 1. Données analytiques des formations du complexe dunaire du Jorf Lasfar.

\begin{tabular}{|c|c|c|c|c|c|c|c|c|c|c|c|c|}
\hline \multirow[t]{2}{*}{ Formations } & \multirow[t]{2}{*}{$\begin{array}{l}\text { Niv. \& } \\
n^{\circ} \text { éch. }\end{array}$} & \multicolumn{4}{|c|}{ Granulométrie } & \multicolumn{3}{|c|}{$\begin{array}{l}\text { Morphoscopie } \\
\text { des quartzs }\end{array}$} & \multicolumn{4}{|c|}{ Analyses physiques et chimiques } \\
\hline & & $\begin{array}{l}M d \\
(\mu m)\end{array}$ & $\begin{array}{l}C d \\
(\mu m) \\
\end{array}$ & $S k$ & So & $\begin{array}{l}N U \\
(\%)\end{array}$ & $\begin{array}{l}E L \\
(\%) \\
\end{array}$ & $\begin{array}{l}R M \\
(\%) \\
\end{array}$ & $\begin{array}{l}\text { MO } \\
(\%) \\
\end{array}$ & $\begin{array}{l}\mathrm{P}_{2} \mathrm{O}_{5} \\
(\%)\end{array}$ & $\begin{array}{l}\mathrm{Fe} \\
(\%)\end{array}$ & $p H$ \\
\hline \multirow{3}{*}{$\begin{array}{l}\text { Paléosol } 4 \\
(0,3 m)\end{array}$} & s4-s & 118 & 20 & 0,85 & 1,24 & 26 & 34 & 40 & 0,32 & 0,14 & 0,64 & 7,90 \\
\hline & $s 4-m$ & 120 & 21 & 0,84 & 1,25 & 27 & 35 & 38 & 0,34 & 0,16 & 0,67 & 8,20 \\
\hline & $s 4-b$ & 124 & 22 & 0,85 & 1,26 & 28 & 37 & 35 & 0,33 & 0,14 & 0,64 & 8,34 \\
\hline $\begin{array}{l}\text { Edif. dunaire } 4 \\
(1,9 m)\end{array}$ & $d 4$ & 124 & 23 & 0,76 & 1,27 & 19 & 39 & 42 & 0,24 & 0,14 & 0,62 & 7,90 \\
\hline Paléosol 3 & s3-s & 112 & 22 & 0,73 & 1,26 & 23 & 32 & 45 & 0,24 & 0,24 & 0,43 & 8,34 \\
\hline$(0,4 m)$ & $s 3-b$ & 119 & 24 & 0,82 & 1,28 & 24 & 33 & 43 & 0,19 & 0,33 & 0,43 & 8,37 \\
\hline $\begin{array}{l}\text { Edif. dunaire } 3 \\
(2,2 m)\end{array}$ & $d 3$ & 178 & 40 & 0,71 & 1,35 & 25 & 34 & 41 & 0,19 & 0,24 & 0,43 & 8,00 \\
\hline Paléosol 2 & $s 2-s$ & 114 & 25 & 0,96 & 1,33 & 21 & 23 & 56 & 0,48 & 0,28 & 0,81 & 7,60 \\
\hline$(0,6 m)$ & $s 2-b$ & 126 & 27 & 0,98 & 1,32 & 23 & 25 & 52 & 0,33 & 0,33 & 0,91 & 7,80 \\
\hline $\begin{array}{l}\text { Edif. dunaire } 2 \\
(3,2)\end{array}$ & $d 2$ & 152 & 28 & 0,72 & 1,35 & 12 & 26 & 62 & 0,19 & 0,48 & 1,15 & 8,00 \\
\hline Paléosol 1 & s1-s & 102 & 22 & 0,96 & 1,26 & 15 & 38 & 47 & 0,28 & 0,67 & 2,11 & 8,10 \\
\hline$(3 m)$ & $s 1-b$ & 116 & 25 & 0,98 & 1,29 & 16 & 39 & 45 & 0,24 & 0,72 & 2,00 & 8,00 \\
\hline Edif. dunaire 1 & $d 1-s$ & 114 & 24 & 0,76 & 1,28 & 19 & 38 & 43 & 0,19 & 0,33 & 1,77 & 8,00 \\
\hline$(2,5 m)$ & $d 1-b$ & 128 & 23 & 0,78 & 1,27 & 17 & 42 & 41 & 0,02 & 0,24 & 1,58 & 8,37 \\
\hline
\end{tabular}

\subsection{La calcimétrie}

La teneur en carbonates est, en moyenne, de $27 \%$ dans les paléosols et de $70 \%$ dans les édifices dunaires. L'enrichissement relatif en carbonates à la base des paléosols prouve l'existence d'un gradient d'accumulation de carbonates dû au lessivage des horizons supérieurs qui ont été érodés. 


\section{Tectonique}

A Jorf Lasfar, le complexe dunaire quaternaire, probablement d'âge Soltanien (Würmien selon la terminologie européenne, TEXIER (1986)), a été affecté d'une série de failles normales et inverses, aussi bien dans le paléosol 1 que dans l'édifice dunaire consolidé le surmontant. Le rejet vertical maximum de ces failles normales peut atteindre $80 \mathrm{~cm}$. Leur direction dominante est N20 et leur pendage varie de $65^{\circ}$ à $75^{\circ}$ vers l'ESE (voir figure 4) (ABOUMARIA et al., 1993; OUADIA et al., 1997).

Dans le but de chercher les relations entre ces failles et les diaclases de la plate-forme d'abrasion et celles du complexe littoral affleurant en falaise vive, nous avons comparé les directions de ces accidents superposés. La direction dominante des diaclases se situe entre N20 et N90 (OUADIA et al., 1997; OUADIA, 1998). Cette coïncidence (N20 mesurée aussi bien dans la plate-forme que dans le complexe dunaire quaternaire) permet de suggérer que l'activité tectonique qui affecte le complexe dunaire quaternaire du Jorf Lasfar peut être la continuation des mouvements anté-quaternaires faisant rejouer les accidents hercyniens de direction dominante N-S dans les Doukkala (GIGOUT, 1951). Ceci est confirmé par les travaux de RUELLAN \& AUZENDE (1984) sur la fracturation ayant affecté le socle du plateau continental des environs d'El Jadida et du Jorf Lasfar puisqu'elle présente une direction dominante comprise entre N20 à N90.

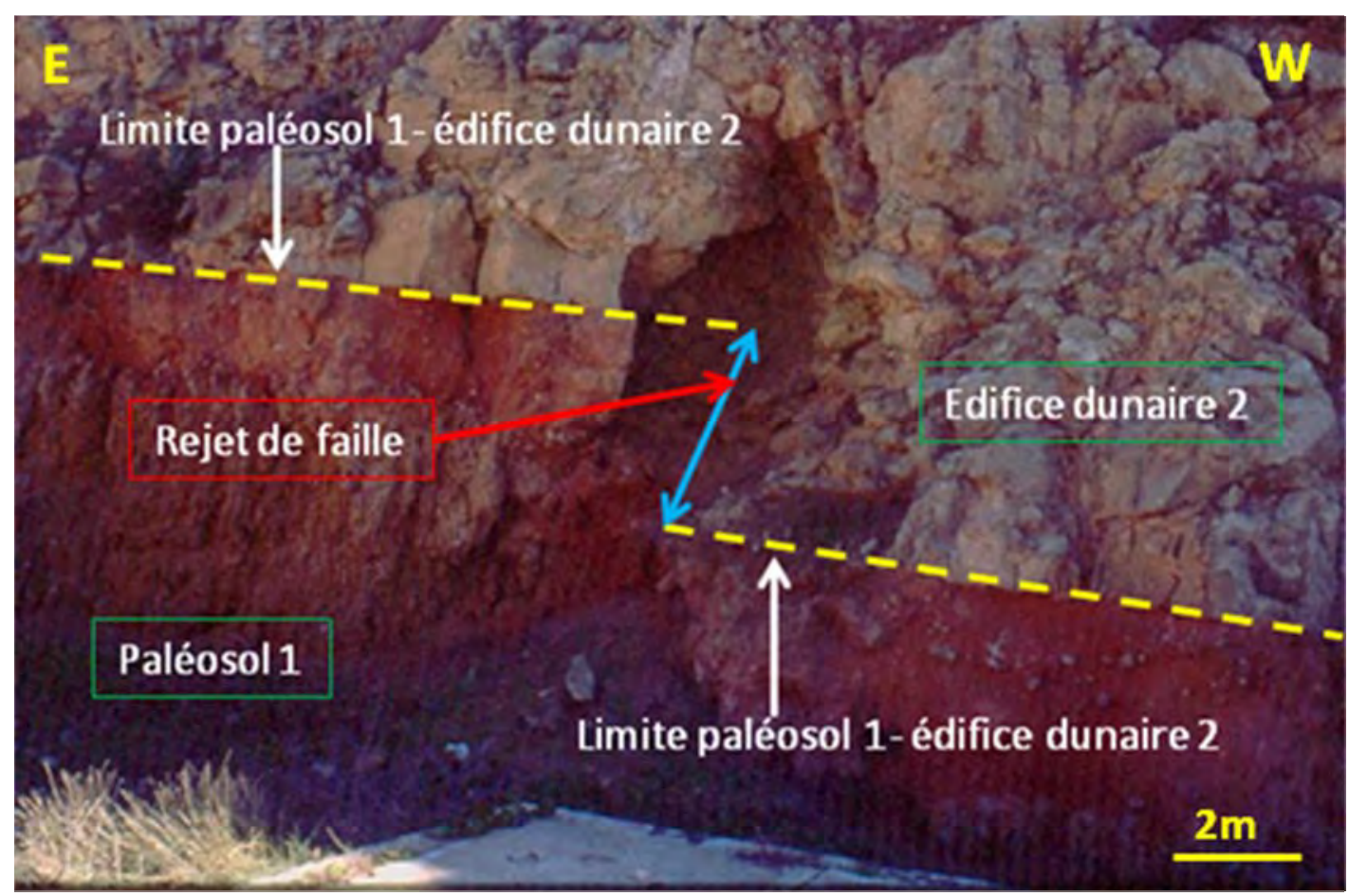

Figure 4. Faille inverse du complexe dunaire du Jorf Lasfar 
Les mouvements néotectoniques du secteur du Jorf Lasfar correspondent à des rejeux de la tectonique hercynienne et alpine. Cette activité néotectonique semble être similaire à celle signalée dans la région de Rabat (MOREL, 1987), dans la région de Rabat-Kénitra (ABERKAN, 1989 ; ABERKAN, 1996), dans la Méséta côtière (ABERKAN, 1993) et dans la région de Larache (ADIL \& ABERKAN, 1993 ; ADIL, 1996). Enfin si GIGOUT (1951) a considéré la Méséta côtière comme un secteur où il n'y a aucun indice de déformation au Quaternaire, les mouvements néotectoniques du Jorf Lasfar (traités dans cet article) remettent en question cette conclusion.

\section{Discussion}

Le complexe quaternaire du Jorf Lasfar est constitué de quatre édifices dunaires consolidés surmontés chacun d'un paléosol dont les horizons supérieurs ont été érodés avant le dépôt éolien suivant.

Les sables fins du complexe sont en général très bien classés, leur taille est moyenne (124 $\mu \mathrm{m}$ en moyenne). Les grains de quartz les plus fréquents dans les édifices dunaires sont les ronds mats (plus de 35\%). Les émoussés luisants viennent en deuxième position (34\% en moyenne), ce qui suggère leur mise en place sous l'action d'une dynamique éolienne à partir d'un matériel subaquatique. En outre, la présence des bioclastes marins en gros fragments montre que ces édifices dunaires se sont formés non loin de la ligne du rivage. La présence, dans les édifices dunaires, des grains de quartz intermédiaires entre les émoussés luisants et les ronds-mats (ronds luisants et émoussés mats) prouve des échanges répétés entre les deux domaines de dynamiques (marine littorale et éolienne). (OUADIA et al., 1993). Des résultats comparables ont été trouvés dans les cordons littoraux du Nord de Rabat (ABERKAN \& LEGIGAN, 1984 ; ABERKAN, 1989), de Larache (ADIL \& ABERKAN, 1993; ADIL, 1996) et de Casablanca (ZANNIBY, 1997).

Le taux des carbonates est généralement supérieur à $60 \%$ avec une abondance de bioclastes marins façonnés par la houle sur la plage puis repris par le vent (bioclastes de taille petite et de forme émoussée). La teneur pondérale faible des minéraux lourds et la valeur plus ou moins importante de leur indice de maturité prouvent une importante altération des apports détritiques (ABERKAN et al., 1982; AKIL \& GAYET, 1988; AKIL, 1990). Le cortège des minéraux lourds est caractérisé par l'abondance de la hornblende issue des formations du massif métamorphique des Réhamna. Le pyroxène vient plus probablement du volcanisme plio-quaternaire des bassins versants drainés par l'Oued Oum Rbia tels que le Moyen Atlas et la Méséta côtière (OUADIA \& ABERKAN, 1996) par l'intermédiaire de la dérive littorale de direction N-S.

Postérieurement à leur mise en place, ces dunes ont subi une action diagenétique avec dissolution et recristallisation des bioclastes et par la transformation de la matrice micritique en ciment, qui constitue une mosaïque de cristaux microsparitiques, 
colmatant les vides intergranulaires, la lithification des édifices dunaires s'effectuant dans le milieu continental sous l'influence des eaux météoriques.

Les paléosols surmontant chacun d'édifice dunaire témoignent un contexte climatique humide. Tous les paléosols sont érodés dans leur partie supérieure et ce suite à une déflation éolienne qui annonce la fin de la phase humide et le début de la phase sèche qui la succède. Cette érosion effectue les paléosols avant leur fossilisation par les apports dunaires qui les surmontent. Le paléosol le plus évolué étant le paléosol 1 (voir figure 2). Le pourcentage relativement élevé de la matière organique et du phosphore assimilable dans ces paléosols serait en relation avec cette activité biologique du sol. Le $\mathrm{pH}$ basique s'expliquerait par la forte teneur des carbonates issus de la dissolution des bioclastes. La légère élévation du taux des carbonates à la base des paléosols ainsi que la présence, en particulier pour le paléosol 1 et 3 , d'une structure polyédrique témoignent aussi d'une accumulation pédogénétique. La teneur en fer du paléosol 1 doit être expliquée par un degré de rubéfaction relativement plus important au niveau de ce paléosol.

Les édifices dunaires du complexe du Jorf Lasfar se sont mis en place au cours d'une période relativement sèche dite soltanienne. Le dépôt de chaque édifice dunaire est contemporain d'un épisode d'aridité au début duquel une déflation des horizons supérieurs du profil pédologique sous-jacent a eu lieu. Cette période est entrecoupée de quatre épisodes climatiques relativement humides qui ont favorisé le développement des paléosols ou de remontée de la nappe aquifère apportant de l’humidité favorable aux zones.

Les formations quaternaires littorales supérieures du Jorf Lasfar enregistrent une activité néotectonique observée particulièrement au niveau du paléosol 1 et de l'édifice dunaire 2.

Nos observations sur les formations du Jorf Lasfar confirment celles, toutes proches, de RUELLAN \& AUZENDE (1984) qui ont montré à partir de l'étude sismique du plateau continental d'El Jadida que la plate-forme d'abrasion calcaire moderne est découpée en une succession de blocs basculés. Ce découpage en blocs se fait suivant un réseau de cassures dont les directions prédominantes sont N20, N90, N120 et N160. La première de ces directions est très proche de celle que nous avons mesurée pour les diaclases observées à la surface de la plate-forme d'abrasion et dans le complexe littoral d'El Jadida taillé en falaise vive (OUADIA et al., 1997). Ceci permet de conclure à une activité tectonique verticale jusqu'à l'Actuel qui se manifeste par une déformation pléistocène à Jorf Lasfar. La néotectonique ayant affecté la zone du Jorf Lasfar n'est pas exceptionnelle sur le littoral atlantique. MOREL et al. (1987) ont pu mettre en évidence, d'après l'analyse microtectonique des fractures et des failles dans la région de Rabat, des mouvements de compression au cours du Pléistocène ancien et probablement moyen de direction NE-SW. Ces mouvements côtiers sont comparables à ceux qui, plus au Nord, sont connus dans le domaine rifain et attribués à une phase de compression à la limite 
Plio-Quaternaire (MOREL et al., 1987). La même activité néotectonique est décrite dans les régions de Rabat-Kénitra (ABERKAN, 1989 ; ABERKAN, 1996) et dans la région de Larache (ADIL \& ABERKAN, 1993; ADIL, 1996). Deux tendances peuvent être dégagées des mouvements néotectoniques du secteur du Jorf Lasfar : les rejets de la tectonique hercynienne et alpine se partagent quant à leurs directions.

\section{Conclusion}

Le complexe dunaire du Jorf Lasfar, représente la coupe la plus détaillée et la plus complète dans la région étudiée, avec quatre édifices dunaires quaternaires consolidés entrecoupés de quatre paléosols développés dont le plus ancien (paléosol 1) semble le plus évolué. Les horizons supérieurs de ces sols fossiles ont été érodés avant leur fossilisation, ce qui indique de profonds changements répétés des conditions climatiques. La mise en place des calcarénites du complexe dunaire du Jorf Lasfar est à attribuer à une dynamique éolienne confirmée par l'abondance des grains de quartz ronds mats malgré la proximité de la plage. La fréquence des grains de quartz de types intermédiaires (ronds luisants et émoussés mats) témoigne de l'interaction des dynamiques marine littorale et éolienne. L'étude des minéraux lourds suggère trois sources possibles des matériaux : le massif des Réhamna, le Moyen Atlas et la Méséta côtière (OUADIA \& ABERKAN, 1996). Le rôle de la dérive littorale de direction N-S n'est pas à négliger puisque l'Oued Oum Rbia débouche dans l'Atlantique $25 \mathrm{~km}$ plus au Nord.

Les conditions paléoclimatiques durant la mise en place du complexe dunaire du Jorf Lasfar, correspondent à une alternance de conditions arides permettant la mise en place des édifices dunaires et de phases tempérées humides au cours desquelles des paléosols rubéfiés se développent.

Les unités les plus anciennes du complexe du Jorf Lasfar ont été affectées par une activité néotectonique qui pourrait être liée à une réactivation de failles hercyniennes ou alpines du substratum. Cette activité néotectonique est comparable à celle signalée dans d'autres zones de la façade atlantique marocaine. La Méséta côtière marocaine dont fait partie le secteur du Jorf Lasfar n'a donc pas été une zone stable au cours du Quaternaire comme cela était décrit auparavant.

\section{Références bibliographiques}

ABERKAN M., AKIL M., GAYET J., LEGIGAN P. (1982). Intérêt lithostratigraphique et paléogéographique de l'altération des minéraux lourds. $107^{\mathrm{ème}}$ Congrès National des Sociétés Savantes, Brest, Sciences, III, pp 213-221.

ABERKAN M., LEGIGAN P. (1984). Les dunes littorales quaternaires dans le Gharb méridional. Etude sédimentologique. Bull. Inst. géol. Bassin d'Aquitaine, Bordeaux, Vol. 35, pp 31-44. 
ABERKAN M. (1989). Etude des formations quaternaires des marges du bassin du Rharb (Maroc nord-occidental). Thèse Doct. es Sc., Bordeaux I, 290 p.

ABERKAN M. (1993). Qui a contrôlé la mise en place des formations quaternaires au Maroc ? Etude d'exemples. $11^{\text {ème }}$ Colloque des bassins sédimentaires marocains, Rabat, $1 \mathrm{p}$.

ABERKAN M. (1996). L'instabilité des formations quaternaires de la côte atlantique marocaine et son impact sur l'aménagement. $13^{\text {ème }}$ Colloque des Bassins sédimentaires marocains, Marrakech, 1 p.

ABOUMARIA K. (1993). Les formations quaternaires du Sahel des Doukkala (Méséta occidentale marocaine): mise en place et évolution post-sédimentaire. Thèse de $3^{\text {ème }}$ cycle, Université Mohammed V, Rabat, 186 p.

ABOUMARIA K., ABERKAN M., OUADIA M. (1993). Aspects sédimentologiques des formations quaternaires du Sahel des Doukkala (Méséta occidentale marocaine). $11^{\text {ème }}$ Colloque des bassins sédimentaires marocains, Rabat, 4 p.

ADIL S., ABERKAN M. (1993). Contribution à l'étude des formations littorales quaternaires de Larache (Nord-Occidental du Maroc). $14^{\text {th }}$ I.A.S. regional meeting of sedimentology, Marrakech, pp 249-249.

ADIL S. (1996). Les formations quaternaires littorales de Larache: Sédimentologie, datations radiochimiques, environnement et aménagement. Thèse de $3^{\text {ème }}$ cycle, Fac. Sci, Rabat, 222 p.

AKIL M., GAYET J. (1988). Evolution des minéraux lourds dans les formations actuelles et plio-quaternaires de plate-forme et du littoral atlantique marocain. Bull. Inst. Géol. Bassin d'Aquitaine, Bordeaux, Vol. 43, pp 153-161.

AKIL M. (1990). Les dépôts quaternaires littoraux entre Casablanca et Cap Beddouza (Méséta côtière marocaine) - Etudes géomorphologiques et sédimentologiques. Thèse Doct. es. Sci., Fac. Sci, Rabat, 417 p.

DUNHAM R.J. (1969). Early vadose silt in Townsend mound (reef), New Mexico. Depositional environments in carbonate rocks. Revue Soc. Econ. Paleont. Miner. sp. publication, n 14, G.M. Friedman Edit., pp 139-181. http://dx.doi.org/10.2110/pec.69.03.0139

GIGOUT M. (1947). Quaternaire du littoral atlantique du Maroc. Les dunes quaternaires du Sahel. Revue C. R. Somm. Soc. Géol., France, Vol. 5, pp 71-73.

GIGOUT M. (1951). Etude géologiques sur la Méséta marocaine occidentale (arrière pays de Casablanca, Mazagan et Safi). Revue Trav. Inst. Sc. Chérifien, 3, et Notes et. et Mém. Serv. Géol., Maroc, 86, 2 t., 507 p.

MOREL J.L., GONORD H., SARTIGES B., ZAMOLO G. (1987). Observations néotectoniques sur le littoral atlantique du Maroc septentrional (région de Rabat): mise en évidence de mouvements compressifs au Pléistocène. Revue Notes Serv. Géol., Maroc, t. 43, Vol. 321, pp 313-319. 
OUADIA M., ABOUMARIA Kh., ABERKAN M. (1993). Nouvelles données sur les formations quaternaires littorales atlantiques entre El Jadida et Safi. $14^{\text {th }}$ I.A.S. regional meeting of sedimentology, Marrakech, $249 \mathrm{p}$.

OUADIA M., ABERKAN M. (1996). Contribution à l'étude géomorphologique et sédimentologique du quaternaire de la vallée de l'Oum Rbia (Méséta côtière atlantique - Maroc). Revue Géologie Méditerranéenne, t. XXIII, Vol. 2, pp 89-99.

OUADIA M., ABERKAN M., ABOUMARIA K. (1997). Les formations quaternaires du littoral atlantique entre Casablanca et Safi: mise en évidence d'une activité néotectonique. 14ème Colloque des bassins sédimentaires marocains, Kénitra, 239 p.

OUADIA M. (1998). Les formations plio-quaternaires dans le domaine mésétien occidental du Maroc entre Casablanca et Safi: Géomorphologie, sédimentologie, paléoenvironnement quaternaires et évolution actuelle. Thèse Doct. es Sc., Fac. Sci., Rabat, n 1646, 319 p.

PURSER B.H. (1980). Sédimentation et diagenèse des carbonates néritiques récents (tome 1). Technip Ed., 366 p.

RUELLAN E., AUZENDE J.M. (1984). Structure et évolution du plateau sous-marin de El Jadida (Mazagan, Ouest Maroc). Bull. Soc. Géol. France (8), t. I, (1), pp 103-114. ZANNIBY F. (1997). Les formations littorales quaternaires de la région de Casablanca: Etudes sédimentologique et géotechnique. Intérêt de la sédimentologie des dépôts côtiers actuels dans l'aménagement de la façade atlantique casablancaise. Thèse de $3^{\text {ème }}$ cycle, Université Hassan II, Ben M'Sik-Casablanca, 227 p. 